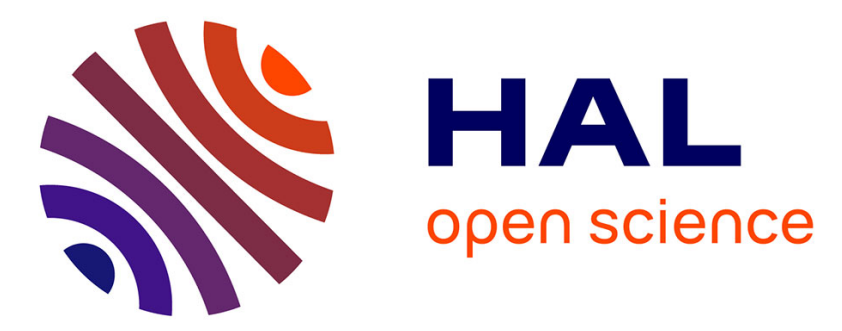

\title{
Methanol as a Sensitive Probe for Spatial and Temporal Variations of the Proton-to-Electron Mass Ratio
}

Paul Jansen, Li-Hong Xu, Isabelle Kleiner, Wim Ubachs, Hendrick Bethlem

\section{To cite this version:}

Paul Jansen, Li-Hong Xu, Isabelle Kleiner, Wim Ubachs, Hendrick Bethlem. Methanol as a Sensitive Probe for Spatial and Temporal Variations of the Proton-to-Electron Mass Ratio. Physical Review Letters, 2011, 106 (10), 10.1103/PhysRevLett.106.100801 . hal-03352662

\section{HAL Id: hal-03352662 https://hal.science/hal-03352662}

Submitted on 23 Sep 2021

HAL is a multi-disciplinary open access archive for the deposit and dissemination of scientific research documents, whether they are published or not. The documents may come from teaching and research institutions in France or abroad, or from public or private research centers.
L'archive ouverte pluridisciplinaire HAL, est destinée au dépôt et à la diffusion de documents scientifiques de niveau recherche, publiés ou non, émanant des établissements d'enseignement et de recherche français ou étrangers, des laboratoires publics ou privés. 


\title{
Methanol as a Sensitive Probe for Spatial and Temporal Variations of the Proton-to-Electron Mass Ratio
}

\author{
Paul Jansen, ${ }^{1}$ Li-Hong Xu, ${ }^{2}$ Isabelle Kleiner, ${ }^{3}$ Wim Ubachs, ${ }^{1}$ and Hendrick L. Bethlem ${ }^{1}$ \\ ${ }^{1}$ Institute for Lasers, Life and Biophotonics, VU University Amsterdam, De Boelelaan 1081, 1081 HV Amsterdam, The Netherlands \\ ${ }^{2}$ Department of Physics and Centre for Laser, Atomic, and Molecular Sciences, University of New Brunswick, \\ Saint John, New Brunswick E2L 4L5, Canada \\ ${ }^{3}$ Laboratoire Interuniversitaire des Systèmes Atmosphériques (LISA), CNRS UMR 7583 et Universités Paris 7 et Paris Est, \\ 61 avenue du Général de Gaulle, 94010 Créteil Cédex, France
}

(Received 21 December 2010; published 11 March 2011)

\begin{abstract}
The 6.7 and $12.2 \mathrm{GHz}$ masers, corresponding to the $5_{1} \rightarrow 6_{0} A^{+}$and $2_{0} \rightarrow 3_{-1} E$ transitions in methanol $\left(\mathrm{CH}_{3} \mathrm{OH}\right)$, respectively, are among the brightest radio objects in the sky. We present calculations for the sensitivity of these and other transitions in the ground state of methanol to a variation of the proton-toelectron mass ratio. We show that the sensitivity is greatly enhanced due to a cancellation of energies associated with the hindered internal rotation and the overall rotation of the molecule. We find sensitivities of $K_{\mu}=-42$ and $K_{\mu}=-33$, for the $5_{1} \rightarrow 6_{0} A^{+}$and $2_{0} \rightarrow 3_{-1} E$ transitions, respectively. The sensitivities of other transitions in the different isotopologues of methanol range from -88 to 330 . This makes methanol a sensitive probe for spatial and temporal variations of the proton-to-electron mass ratio.
\end{abstract}

PRL 106, 100801 (2011)

PHYSICAL REVIEW LETTERS

DOI: 10.1103/Phy sRevLett.106.100801

A possible spatial or temporal variation of the fine structure constant $\alpha$ or the proton-to-electron mass ratio $\mu$ can be detected by comparing transitions in atoms and molecules as a function of time and/or position. As these transitions have, in general, a different dependence on $\alpha$ and $\mu$, a possible variation might be observed as a shift of the transition frequencies [1,2]. In astrophysical searches, the frequencies of two or more atomic and molecular absorptions from distant objects are compared with the frequencies as measured in the laboratory. This allows one to determine the apparent redshift-and hence distance to the object-and a possible variation. In this Letter, we discuss the use of molecules to detect a variation of $\mu$. The sensitivity of an experiment searching for a frequency shift $\Delta \nu$ due to the variation of $\mu$ depends both on the size of the shift, i.e., the inherent sensitivity of the molecular transition, and on the ability to measure this shift. As a measure for the inherent sensitivity of a transition, the sensitivity coefficient $K_{\mu}$ is defined via

$$
\frac{\Delta \nu}{\nu}=K_{\mu} \frac{\Delta \mu}{\mu} .
$$

For a sensitive test, one needs molecular transitions that are observed with a good signal to noise and that exhibit high $K_{\mu}$. Transitions in hydrogen, the most abundant molecule in outer space and observed at high redshift $(z=3)$, have sensitivities that range from $K_{\mu}=-0.02$ to +0.05 [2-4]. Rotational transitions in CO, the second most abundant molecule in space, have a sensitivity of $K_{\mu}=-1$. The inversion frequency in ammonia is exponentially dependent on $\mu$. As a consequence, the inversion frequency in ammonia has a sensitivity of $K_{\mu}=-4.2$ [5-7].
It was noticed by Flambaum [8], Flambaum and Kozlov [9], DeMille et al. [10], and Bethlem and Ubachs [11] that the sensitivity to a variation of $\alpha$ and $\mu$ is enhanced when an accidental degeneracy occurs between levels which have substantially different dependences on $\alpha$ and $\mu-$ for instance, when rotational splittings are equal to a hyperfine structure splitting [8] or fine structure splitting [11] or when a vibrational splitting is equal to a fine structure splitting [9] or an electronic splitting [10]. However, the molecular transitions discussed in these papers are unlikely to be observed in interstellar objects. In this Letter, we show that a significant enhancement occurs in methanol due to a cancellation of energies associated with the hindered internal rotation and the overall rotation of the molecule. This is particularly relevant as methanol is abundantly present in the Universe.

Methanol is one of the simplest molecules that exhibits hindered internal rotation and has, therefore, been the subject of numerous experimental and theoretical studies [12-18]. The structure of methanol, depicted on the righthand side of Fig. 1, consists of a methyl group, with a hydroxyl group attached. The center of mass of the $\mathrm{OH}$ group is slightly displaced from the symmetry axis of the methyl group. The overall rotation is described by three rotational constants $A, B$, and $C$, associated with the moments of inertia $I_{a}, I_{b}$, and $I_{c}$, respectively, along the three principal axes. The total angular momentum of the molecule is given by the quantum number $J$, while the projection of $J$ onto the molecule fixed axis is given by $K$.

In addition to the overall rotation, methanol has internal rotation; the $\mathrm{OH}$ group rotates with respect to the methyl group. The angle between the two parts of the molecule is denoted by $\gamma$. The rotation is severely hindered, due to the 


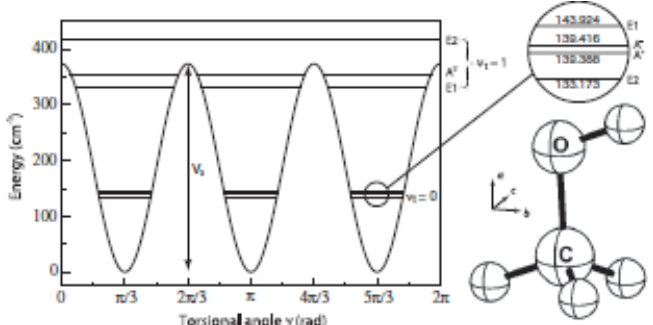

FG. 1. Variation of the potential energy of methanol with the relative rotation $\gamma$ of the $\mathrm{OH}$ group with respect to the methyl group about the molecular axis. Shown are the $J=1,|K|=1$ energies of the lowest torsion-vibrational levels. The splitting between the different symmetry levels is due to tunneling through the potential barriers. The $A$-symmetry species are split further due to the asymmetry of the molecule ( $K$ splitting).

repulsion of a threefold torsional potential energy curve, shown on the left-hand side of Fig. 1. The vibrational levels in this well are denoted by $\nu_{t}$. In lowest order-neglecting higher orders in the potential and centrifugal distortionsthe Hamiltonian can be written as [12]

$$
\begin{gathered}
H=\frac{1}{2} \frac{P_{a}^{2}}{I_{a}}+\frac{1}{2} \frac{P_{b}^{2}}{I_{b}}+\frac{1}{2} \frac{P_{c}^{2}}{I_{c}}+\frac{1}{2} \frac{1}{I_{\mathrm{red}}} p_{\gamma}^{2}+\frac{1}{2} V_{3}(1-\cos 3 \gamma) \\
\text { with } I_{\mathrm{red}}=\frac{I_{a 1} I_{a 2}}{I_{a}}
\end{gathered}
$$

The first three terms describe the overall rotation around the $a, b$, and $c$ axis, respectively. The fourth term describes the internal rotation around the $a$ axis, with $I_{\text {red }}$ the reduced moment of inertia along the $a$ axis, $I_{a 2}$ the moment of inertia of the methyl group along its own symmetry axis, and $I_{a 1}$ the part of $I_{a}$ that is attributed to the $\mathrm{OH}$ group; $I_{a 1}=$ $I_{a}-I_{a 2}$. The fifth term is the lowest order term arising from the torsional potential. If the torsional potential were infinitely high, the threefold barrier would result in three separate harmonic potentials, with rotational

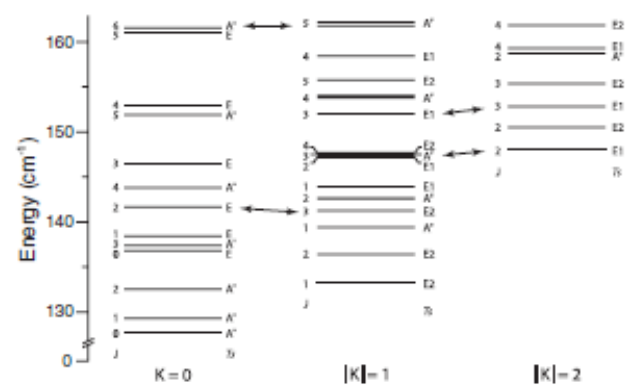

FIG. 2. Energy of the lowest rotational levels in the torsionvibrational ground state $\left(\nu_{t}=0\right)$ of methanol [18]. The levels are denoted by $J$ and $K$ and by their torsional symmetry $T s$. energies corresponding to the harmonic oscillator eigenvalues. In the case of a finite barrier, quantum-mechanical tunneling mixes the levels in the different wells of the potential. As a result, every $K$ level is split into three levels, which can be labeled according to torsional symmetry, $T s$, as $A, E 1$, or $E 2$. Following Lees [14], $E 1$ and $E 2$ symmetries are labeled by the sign of $K$; i.e., levels with $E 1$ symmetry are denoted by a positive $K$ value, whereas levels with $E 2$ symmetry are denoted by a negative $K$ value. For $K \neq 0, A$ levels are further split into + and - components by molecular asymmetry. For $K=0$, only single $E$ and $A^{+}$levels exist. The tunneling motion in the torsional potential is similar to the inversion motion in ammonia, which exhibits an increased sensitivity to a possible variation of the protonto-electron mass ratio $[5,6]$. This similarity prompted us to study the methanol spectrum. In Fig. 2, the lowest energy levels of ${ }^{12} \mathrm{CH}_{3}^{16} \mathrm{OH}$ are shown.

The splitting between the different symmetry levels is related to the tunneling frequency between the different torsional potential wells, and, hence, it is very sensitive to the reduced moment of inertia. In Fig. 3, the energy splittings between $A$ and $E$ levels of the $J=0, K=0$ and the energy splitting between $E 1$ and $E 2$ levels in the $J=1$, $|K|=1$ are plotted as a function of the reduced moment of inertia $I_{\text {red }}$ along the $a$ axis for six different isotopologues of methanol [18]. In analogy with ammonia [5], we expect the tunneling splitting to follow the formula

$$
W_{\gamma}=\frac{a_{0}}{\sqrt{I_{\text {red }}}} e^{-a_{1} \sqrt{I_{\text {mad }}}},
$$

with $W_{\gamma}$ the energy splitting due to hindered rotation and $a_{0}$ and $a_{1}$ constants. The fitted lines in Fig. 3 correspond to $a_{0}=316$ and $433 \mathrm{~cm}^{-1}\left(\mathrm{amu} \AA^{2}\right)^{1 / 2}$ and $a_{1}=4.9$ and $5.1\left(\text { amu } \AA^{2}\right)^{-1 / 2}$ for $K=0$ and $|K|=1$, respectively.

If we assume that possible variations of the proton and neutron masses scale in a similar manner, the reduced

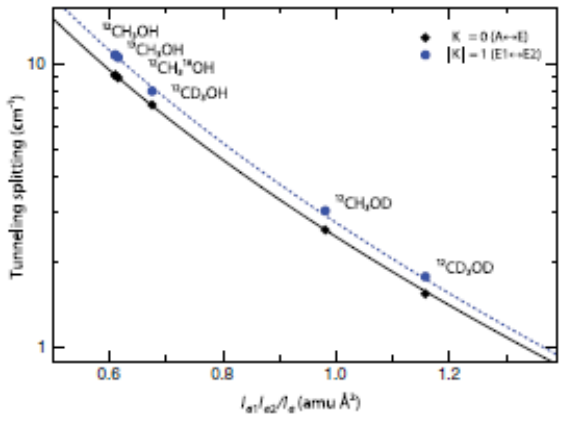

FIG. 3 (color online). Energy splitting between $A$ and $E$ levels of the $J=0, K=0$ (diamonds) and the energy splitting between $E 1$ and $E 2$ levels of the $J=1,|K|=1$ (circles) as a function of the reduced moment of inertia along the $a$ axis for six different isotopologues of methanol [18]. The solid and dashed lines are fits according to Eq. (3). 
moment of inertia is directly proportional to $\mu$. Thus, the sensitivity coefficient is given by

$$
K_{\mu}^{\mathrm{tars}}=\frac{I_{\mathrm{red}}}{W_{\gamma}} \frac{\partial W_{\gamma}}{\partial I_{\mathrm{red}}} .
$$

From the above fits, we find for the $K=0$ and $|K|=1$ splitting a sensitivity coefficient of $K_{\mu}^{\text {tors }}=-2.4$ and $K_{\mu}^{\mathrm{k} s}=-2.5$, respectively.

In the case of ammonia, the tunneling frequency can be directly measured by inducing a transition between the two inversion levels. In the case of methanol, however, the different torsional levels of a specific $J, K$ state have different torsional symmetries, and transitions between these levels are not allowed. Hence, we look for rotational transitions between levels which differ in $K$ and/or $J$. The sensitivity coefficients of these transitions are given by

$$
\begin{aligned}
& K_{\mu}\left(\nu_{t}^{g}, J^{\prime \prime}, K^{\prime \prime}, T s^{\prime \prime} \rightarrow \nu_{t}^{\prime}, J^{\prime}, K^{\prime}, T s^{\prime}\right) \\
& =\frac{\mu(\partial E / \partial \mu)_{\nu_{t}^{\prime \prime}, f^{\prime \prime}, K^{\prime \prime}, T s^{\prime \prime}}-\mu(\partial E / \partial \mu)_{\nu_{f}^{\prime}, J^{\prime}, K^{\prime}, T s^{\prime}}}{E\left(\nu_{t}^{\prime \prime}, J^{\prime \prime}, K^{\prime \prime}, T s^{\prime \prime}\right)-E\left(\nu_{t}^{\prime}, J^{\prime}, K^{\prime}, T s^{\prime}\right)},
\end{aligned}
$$

with

$$
\mu\left(\frac{\partial E}{\partial \mu}\right)_{v_{g}, J, K, T s}=K_{\mu}^{\mathrm{vib}} E_{\mathrm{vib}}+K_{\mu}^{\mathrm{rot}} E_{\mathrm{r \alpha}}+K_{\mu}^{\mathrm{tors}} E_{\mathrm{wors}}
$$

where $K_{\mu}^{\mathrm{vab}}, K_{\mu}^{\mathrm{r \alpha}}$, and $K_{\mu}^{\mathrm{tax}}$ are the sensitivities to a possible variation of the proton-to-electron mass ratio of a vibrational, rotational, and torsional energy level, respectively, In the first-order approximation, $K_{\mu}^{\mathrm{vib}}=-\frac{1}{2}$ and $K_{\mu}^{\mathrm{ro}}=-1$. Equation (5) suggests that the sensitivity is enhanced for transitions between near degenerate levels. In Fig. 2, some of these transitions have been indicated. Let us, for instance, look at the $5_{1} \rightarrow 6_{0} A^{+}$transition, which corresponds to the strong $67 \mathrm{GHz}$ maser line that is observed in many interstellar objects. Using the known energies of the rotational levels and $K_{\mu}^{\text {tas }}=-2.4$, we find $K_{\mu}=-41$. Similarly, for the $2_{0} \rightarrow 3_{-1} E$ transition, which corresponds to the $12.2 \mathrm{GHz}$ maser line, we find $K_{\mu}=-33$

In order to validate this simple model, we have simulated the methanol level energies by using BELGI [19]. This code was modified and improved by Xu et al. in a number of ways useful for treating the large data sets available for the methanol molecule [18]. Note that, rather than the Hamiltonian given in Eq. (2), BELGI uses an axis transformation (the so-called "rho-axis method") to simplify the calculation. This Hamiltonian is diagonalized in a twostep process to obtain the level energies. By using a set of 119 molecular constants for ${ }^{12} \mathrm{CH}_{3}^{16} \mathrm{OH}$ and 53 or more for the other isotopologues taken from Ref. [18], the lower energy levels are found typically with an accuracy $<50 \mathrm{kHz}$. In order to obtain the sensitivity to a variation of the proton-to-electron mass ratio of each level, the molecular constants are scaled by using the relations given in Ref. [20].

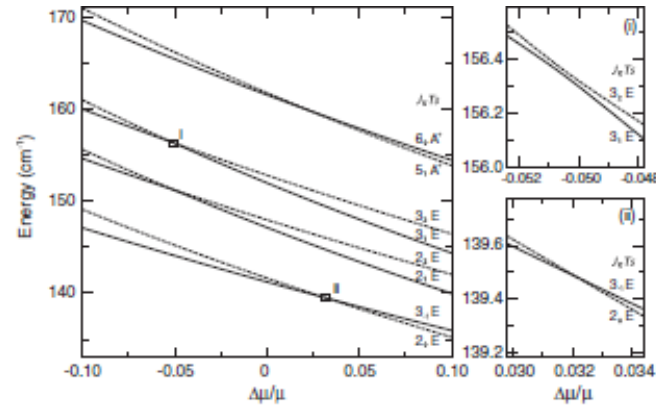

FIG. 4. Energies of selected rotational levels as a function of the fractional change in the proton-to-electron mass ratio. The panels on the right-hand side of the figure show enlargements of selected crossings. It is seen that levels with the same $J$ give rise to avoided crossings, whereas levels with different $J$ do not interact.

In Fig. 4, the energies of selected states (indicated in Fig. 2) are shown as a function of the fractional change of the proton-to-electron mass ratio. As expected, the energies of all rotational levels decrease for larger values of $\Delta \mu / \mu$. However, levels that have different $J$ and $K$ are seen to have a different dependence. The sensitivity coefficient for a transition connecting different levels is given by the difference of the slopes divided by their energy difference-in accordance with Eq. (5). Large sensitivity coefficients are expected when different levels cross in the vicinity of $\Delta \mu / \mu=0$. If the levels cross at $\Delta \mu / \mu<0$, $K_{\mu}$ will be positive; if the levels cross at $\Delta \mu / \mu>0, K_{\mu}$ will be negative. Note that the behavior of the sensitivity coefficient very close to the resonance depends on the quantum numbers of the levels involved. For equal $J$, the levels will mix and $K_{\mu}$ goes to zero, whereas if the levels have unequal $J, K_{\mu}$ goes to infinity.

We have calculated the sensitivity coefficients for several transitions in the most common isotopologues of methanol. The most sensitive transitions involving levels with $J \leq 10$ are listed in Table I. The error in the last digit of the $K_{\mu}$ coefficients is quoted in brackets. The error has 3 sources: (i) errors due to the uncertainty in the determination of the molecular constants, (ii) errors due to inexactness of the scaling relations of higher order constants, and (iii) errors due to neglecting the $\mu$ dependence of the torsional potential. We estimate the error to be below $5 \%$ when $K_{\mu} \geq 1$ or below 0.05 when $K_{\mu}<1$ (for further details, see [20]). It is seen that the values of the sensitivity coefficients for the $5_{1} \rightarrow 6_{0} A^{+}$and $2_{0} \rightarrow 3_{-1} E$ transitions agree well with those found by using Eq. (5). In Ref. [20], the sensitivity coefficients for all transitions that have been observed in interstellar masers [17] are listed.

It is important to note that the sensitivity coefficients of the transitions in Table I have both large positive and large negative values; i.e., if the proton-to-electron mass ratio 
TABLE I. Selected transitions and $K_{\mu}$ coefficients for different isotopologues of methanol, calculated with BELGI. All lines listed for ${ }^{12} \mathrm{CH}_{3}^{16} \mathrm{OH}$ are observed as maser lines [17], except for the two lines marked with an asterisk. The error in the last digit(s) is quoted within brackets.

\begin{tabular}{|c|c|c|c|}
\hline Isotopologue & Transition, $J_{K}$ & Transition $(\mathrm{MHz})$ & $K_{\mu}$ \\
\hline \multirow[t]{18}{*}{${ }^{12} \mathrm{CH}_{3}^{16} \mathrm{OH}$} & $1_{1} \rightarrow 1_{1} A^{\mp}$ & 834.280 & $-1.03(5)$ \\
\hline & $3_{1} \rightarrow 3_{1} A^{\mp}$ & 5005.302 & $-1.03(5)$ \\
\hline & $5_{1} \rightarrow 6_{0} A^{+}$ & 6668.567 & $-42 .(2)$ \\
\hline & $9_{-1} \rightarrow 8_{-2} E$ & 9936.137 & $11.5(6)$ \\
\hline & $5_{2} \rightarrow 4_{3} A^{+}$ & $9978.718^{*}$ & 53.(3) \\
\hline & $5_{2} \rightarrow 4_{3} A^{-}$ & $10058.295^{*}$ & $52 .(3)$ \\
\hline & $2_{0} \rightarrow 3_{-1} E$ & 12178.587 & $-33 .(2)$ \\
\hline & $2_{1} \rightarrow 3_{0} E$ & 19967.376 & $-5.9(3)$ \\
\hline & $9_{2} \rightarrow 10_{1} A^{+}$ & 23120.916 & $-11.7(6)$ \\
\hline & $3_{2} \rightarrow 3_{1} E$ & 24928.726 & 17.9(9) \\
\hline & $2_{2} \rightarrow 2_{1} E$ & 24934.399 & $17.9(9)$ \\
\hline & $8_{2} \rightarrow 9_{1} A^{-}$ & 28969.965 & $-9.5(6)$ \\
\hline & $4_{-1} \rightarrow 3_{0} E$ & 36169.259 & $9.7(5)$ \\
\hline & $6_{2} \rightarrow 5_{3} A^{-}$ & 38293.273 & $-15.1(8)$ \\
\hline & $6_{2} \rightarrow 5_{3} A^{+}$ & 38452.632 & $-15.0(8)$ \\
\hline & $7_{0} \rightarrow 6_{1} A^{+}$ & 44069.364 & 5.2(3) \\
\hline & $1_{0} \rightarrow 2_{-1} E$ & 60531.477 & $-7.4(4)$ \\
\hline & $1_{1} \rightarrow 2_{0} E$ & 68305.629 & $-2.4(1)$ \\
\hline \multirow[t]{8}{*}{${ }^{12} \mathrm{CD}_{3}^{16} \mathrm{OH}$} & $1_{1} \rightarrow 2{ }_{2} E$ & 1202.296 & $330 .(20)$ \\
\hline & $2_{0} \rightarrow 1_{1} E$ & 1424.219 & $-42 .(2)$ \\
\hline & $9_{2} \rightarrow 8_{3} E$ & 2827.262 & 43.(2) \\
\hline & $5_{1} \rightarrow 6_{0} A^{+}$ & 2971.067 & 93.(5) \\
\hline & $4_{-1} \rightarrow 3_{0} E$ & 4011.340 & $-73 .(4)$ \\
\hline & $6_{4} \rightarrow 5_{5} E$ & 6723.841 & 167.(8) \\
\hline & $9_{6} \rightarrow 10_{5} A^{+}$ & 20088.522 & $-88 .(4)$ \\
\hline & $9_{6} \rightarrow 10_{5} A^{-}$ & 20088.522 & $-88 .(4)$ \\
\hline \multirow[t]{2}{*}{${ }^{12} \mathrm{CH}_{3}^{18} \mathrm{OH}$} & $9_{2} \rightarrow 10_{1} A^{-}$ & 2604.912 & 93.(5) \\
\hline & $2_{0} \rightarrow 3_{-1} E$ & 11629.591 & $-34 .(2)$ \\
\hline \multirow[t]{2}{*}{${ }^{12} \mathrm{CD}_{3}^{16} \mathrm{OD}$} & $1_{0} \rightarrow 1_{-1} E$ & 2237.883 & 45.(2) \\
\hline & $7_{-4} \rightarrow 8_{-3} E$ & 2329.088 & $-80 .(4)$ \\
\hline${ }^{13} \mathrm{CH}_{3}^{16} \mathrm{OH}$ & $9_{-1} \rightarrow 8_{-2} E$ & 1989.502 & $-63 .(3)$ \\
\hline
\end{tabular}

varies, some transitions will shift to higher frequencies while others shift to lower frequencies. This makes it possible to perform a test of the variation of the protonto-electron mass ratio by using transitions in methanol only, thus avoiding the many systematic effects that plague tests based on comparing transitions in different molecules. Detection of methanol at even modest redshift will facilitate more stringent bounds on a possible variation of $\mu$ over cosmological time scales. Darling et al. [16] have performed a dedicated survey for $6.7 \mathrm{GHz}$ methanol maser emission in $\mathrm{OH}$ megamaser galaxies at $0.11<z<0.27$. No detections were made in the 25 observed objects. It is our hope that this Letter will stimulate broader surveys.

The high sensitivity coefficients in methanol are also beneficial for probing variation of $\mu$ as a result of chameleonlike scalar fields. These fields predict a dependence of $\mu$ on the local matter density. Recently, Levshakov et al.
[21] compared ammonia spectra taken at high (terrestrial) and low (interstellar) densities of baryonic matter and observed a statistically significant variation of $\mu$. Using methanol, rather than ammonia, in these tests should result in an at least 1 order of magnitude increase in sensitivity.

Furthermore, methanol is a promising candidate for laboratory tests on a possible variation of $\mu$. For instance, when combined, the $1_{1} \rightarrow 2_{1} E$ transition and the $4_{-1} \rightarrow$ $3_{0} E$ transition in $\mathrm{CD}_{3} \mathrm{OH}$ have a sensitivity that is more than 400 times larger than a pure rotational transition. Note that the large dipole moment and low mass make it possible to manipulate methanol molecules by using electric fields.

This research has been supported by NWO via a VIDI grant and by the ERC via a Starting grant. L.-H. X. gratefully acknowledges financial support from the Natural Science and Engineering Research Council of Canada.

Note added.-Recently, Kozlov and Levshakov [22] reported an enhanced $K_{\mu}$ for specific transitions in $\mathrm{H}_{3} \mathrm{O}^{+}$.

[1] J. K. Webb et al., Phys. Rev. Lett. 82, 884 (1999).

[2] E. Reinhold et al., Phys. Rev. Lett. 96, 151101 (2006).

[3] R. I. Thompson, Astrophys. Lett. 16, 3 (1975).

[4] D. A. Varshalovich and S.A. Levshakov, JETP Lett. 58, 237 (1993).

[5] J. van Veldhoven et al., Eur. Phys. J. D 31, 337 (2004).

[6] V. V. Flambaum and M.G. Kozlov, Phys. Rev. Lett. 98, 240801 (2007).

[7] M. T. Murphy, V. V. Flambaum, S. Muller, and C. Henkel, Science 320, 1611 (2008).

[8] V. V. Flambaum, Phys. Rev. A 73, 034101 (2006).

[9] V. V. Flambaum and M.G. Kozlov, Phys. Rev. Lett. 99, 150801 (2007)

[10] D. DeMille et al., Phys. Rev. Lett. 100, 043202 (2008).

[11] H. L. Bethlem and W. Ubachs, Faraday Discuss. 142, 25 (2009).

[12] C. C. Lin and J. D. Swalen, Rev. Mod. Phys. 31, 841 (1959).

[13] B. Kirtman, J. Chem. Phys. 37, 2516 (1962).

[14] R. M. Lees, Astrophys. J. 184, 763 (1973).

[15] E. Herbst, J. K. Messer, F. C. D. Lucia, and P. Helminger, J. Mol. Spectrosc. 108, 42 (1984).

[16] J. Darling, P. Goldsmith, D. Li, and R. Giovanelli, Astron. J. 125, 1177 (2003).

[17] H. S. P. Müller, K. M. Menten, and H. Mäder, Astron. Astrophys. 428, 1019 (2004).

[18] L.-H. Xu et al., J. Mol. Spectrosc. 251, 305 (2008), and references therein.

[19] J. T. Hougen, I. Kleiner, and M. Godefroid, J. Mol. Spectrosc. 163, 559 (1994) (available through http// www.ifpan.edu.pl/ kisiel/introt/introt.htm\#belgi).

[20] See supplemental material at http://link.aps.org/ supplemental/10.1103/PhysRevLett.106.100801 for further details on the calculations and a more extensive list of $K_{\mu}$ coefficients.

[21] S.A. Levshakov et al., Astron. Astrophys. 512, A44 (2010).

[22] M. G. Kozlov and S. A. Levshakov, Astrophys. J. 726, 65 (2011). 